\title{
Finite-size scaling as a tool in the search for the QCD critical point in heavy ion data
}

\author{
Eduardo S. Fraga ${ }^{1 *}$, Letícia F. Palhares ${ }^{1,2 \dagger}$ and Paul Sorensen ${ }^{3 \ddagger}$ \\ ${ }^{1}$ Instituto de Fúsica, Universidade Federal do Rio de Janeiro, \\ Caixa Postal 68528, Rio de Janeiro, RJ 21941-972, Brazil \\ ${ }^{2}$ Institut de Physique Théorique, CEA/DSM/Saclay, \\ Orme des Merisiers, 91191 Gif-sur-Yvette cedex, France \\ ${ }^{3}$ Physics Department, Brookhaven National Laboratory, Upton, NY 11973-5000, USA
}

\begin{abstract}
Given the short lifetime and the reduced volume of the quark-gluon plasma (QGP) formed in high-energy heavy ion collisions, a possible critical endpoint (CEP) will be blurred in a region and the effects from criticality severely smoothened. Nevertheless, the non-monotonic behavior of correlation functions near criticality for systems of different sizes, given by different centralities in heavy ion collisions, must obey finite-size scaling. We apply the predicting power of scaling plots to the search for the CEP of strong interactions in heavy ion collisions using data from RHIC and SPS. The results of our data analysis exclude a critical point below chemical potentials $\mu \sim 450 \mathrm{MeV}$. Extrapolating the analysis, we speculate that criticality could appear slightly above $\mu \sim 500 \mathrm{MeV}$. Using available data we extrapolate our scaling curves to predict the behavior of new data at lower center-of-mass energy, currently being investigated in the Beam Energy Scan program at RHIC. If it turns out that the QGP phase is no longer achievable in heavy ion experiments before the CEP is reached, FSS might be the only way to experimentally estimate its position in the phase diagram.
\end{abstract}

High-energy heavy ion collisions can produce energy densities that are high enough to generate a new state of matter, the quark-gluon plasma (QGP), probing a new region in the phase diagram of strong interactions and testing quantum chromodynamics (QCD) under very extreme conditions. These experimental events are often referred to as little bangs since they reproduce some of the conditions found in the early universe at the time of the primordial quark-hadron transition. Nevertheless, one should bear in mind that the space-time scales that set the dynamics of the quark-gluon plasma formed in heavy ion collisions differ from the cosmological ones by almost twenty orders of magnitude. This means that the early universe at the time of the QCD phase transition(s) was already very large, in the sense that a description assuming the thermodynamic limit is well justified, whereas in the case of heavy ion collisions one should be more cautious.

In fact, even if this issue is usually overlooked, it has been shown using lattice simulations [1, 2] and different effective model approaches [3-6] that finite-size effects can dramatically modify the phase structure of strong interaction, dislocating critical lines and critical points, and also affect significantly the dynamics of phase conversion [7, 8]. Moreover, as discussed in Ref. [6], the modifications suffered by the phase diagram features, such as the critical line and isentropic trajectories, are sensitive to the boundary conditions, as expected for small systems. Therefore, the data corresponding to small QGP systems, such as the ones formed in non-central heavy ion colli-

\footnotetext{
*fraga@if.ufrj.br

†leticia@if.ufrj.br

${ }_{\ddagger}^{\ddagger}$ psoren@bnl.gov
}

sions, should not be naively compared to what one would expect in the thermodynamic limit. A direct consequence of this fact is that all signatures of the second-order critical endpoint based on the non-monotonic behavior 9, 10] or sign modifications [11] of particle correlation fluctuations will probe a pseudocritical endpoint that can be significantly shifted from the genuine (unique) critical endpoint by finite-size corrections and will be sensitive to boundary effects. This feature, together with the even more crucial limitation on the growth of the correlation length due to the finite (short) lifetime of the plasma state and critical slowing down [12, 13], makes the experimental searches of signatures of the presence of a critical point at lower energies very challenging, even if one considers higher moments which grow faster with the correlation length as suggested in Ref. [10]. The rounding and smoothening of fluctuation peaks tend to hide them behind the background. Besides, measuring higher moments of fluctuations of experimental observables in heavy-ion collisions is also a challenging task.

On the other hand, given the fact that the thermal environment corresponding to the region of QGP formed in heavy ion collisions can be classified according to the centrality of the collision, so that events can be separated according to the size of the plasma that is created, the finiteness of the system also brings a bright side: the possibility of finite-size scaling (FSS) analysis [14 16] (For reviews, see Refs. [19 21]). FSS is a powerful statistical mechanics technique that prescinds from the knowledge of the details of a given system; instead, it provides information about its criticality based solely on very general characteristics. Although it is clearly not simple to define an appropriate scaling variable in the case of heavy ion collisions, the flexibility of the FSS method allows for a pragmatic approach for the use of scaling plots in 
the search for the critical endpoint as was delineated in Ref. [6]. The essential point is that although the reduced volume of the QGP formed in high-energy heavy ion collisions will dissolve a possible critical point into a region and make the effects from criticality severely smoothened, the non-monotonic behavior of correlation functions for systems of different sizes, given by different centralities, must obey FSS near criticality 19 21.

In this paper we apply the predicting power of scaling plots to the search for the CEP of strong interactions in heavy ion collisions using data from RHIC and SPS. Defining appropriate scaling variables, we generate scaling plots for data sets with $\sqrt{s_{N N}}=$ 17.3, 19.6, 62.4, 130, $200 \mathrm{GeV}$. From the results of our data analysis we can exclude a critical point below chemical potentials $\mu \sim 450 \mathrm{MeV}$. This seems to be consistent with lattice results that exclude a chiral critical point in the region of $\mu$ smaller or comparable to the temperature [17].

Extrapolating the analysis, we speculate that criticality could appear slightly above $\mu \sim 500 \mathrm{MeV}$. Using available data we extrapolate our scaling curves to predict the behavior of new data at lower center-of-mass energy, currently being investigated in the Beam Energy Scan program at RHIC. If it turns out that the QGP phase is no longer achievable in heavy ion experiments before the CEP is reached, FSS might be the only way to experimentally estimate its position in the phase diagram.

Finding the appropriate scaling variable(s) is almost never an easy exercise for real physical systems. In this analysis we pragmatically choose to work with a onedimensional scaling function motivated by the simplification of thermal model descriptions of the freeze-out region [18], which connects temperature and chemical potential, so that our scaling variable can be built from $\sqrt{s_{N N}}$.

It is well known that as one goes near criticality in a second-order phase transition the correlation functions of the system scale with some power of the correlation length. This feature is independent of the details of the system under consideration, and the power is dictated by which correlation function one considers and the universality class to which the system belongs [19, 20]. Therefore, it is clear that observables that can be written in terms of the correlation functions will exhibit a nonmonotonic behavior near a critical point, the effect being more dramatic as one goes up in moments of the fluctuations. This behavior provides a clean evidence of the presence of a critical point [19, 20]. Thus, it could be used as a precise signature in the analysis of data from heavy ion collisions using event-by-event analysis, as proposed in Refs. [9, 10], were it not the case that these systems are usually very small and come in different sizes. Therefore, the peaks that are typical from this divergent behavior in the thermodynamic limit will be smoothened by the effects of finite size and short lifetime of the systems un- der consideration in heavy ion collisions. Even higher moments [10], which could provide stronger divergences (that grow with higher powers of the correlation length) are more difficult to analyze under these circumstances, from the experimental point of view. So, as an alternative in data analysis, besides looking for non-monotonicity in particle multiplicities, one should also test for the scaling that differently-sized systems must obey near criticality, i.e. finite-size scaling. This also corresponds to the correct way to extrapolate the results of measurements of the finite system to the thermodynamic limit.

The FSS hypothesis 14] was conjectured before the development of the renormalization group (RG). However, it can be derived quite naturally by applying RG techniques to critical phenomena [16, 21]. For a system with typical linear dimension $L$, the singular part of the free energy density scales as

$$
f_{s}\left(\{g\}, L^{-1}\right)=\ell^{-d} f_{s}\left(\left\{g^{\prime}\right\}, \ell L^{-1}\right),
$$

when the lengths are reduced by a factor $\ell$ in a given RG transformation. $\{g\}$ are couplings and $d$ is the space dimension. The fact that the system is finite is irrelevant for this implementation, since the RG transformations are local. Near a RG fixed point, one can write (1) in terms of the right eigenvectors of the linearized RG transformation:

$$
f_{s}\left(t, r, \ldots, L^{-1}\right)=\ell^{-d} f_{s}\left(t \ell^{y_{t}}, r \ell^{y_{r}} \ldots, \ell L^{-1}\right),
$$

so that one notices that $L^{-1}$ behaves like a relevant eigenvector with eigenvalue $\Lambda_{L}=\ell$, and $y_{L}=1$. Here, $t=\left(T-T_{c}\right) / T_{c}$ is the reduced temperature, $T_{c}$ being the temperature associated with the critical point. The reduced temperature $t$ is a dimensionless measure of the distance to the critical point when no other external parameter is considered. In the presence of other external parameters, such as the baryonic chemical potential $\mu$, one should redefine this distance accordingly to include the dependence on $r=\left(\mu-\mu_{c}\right) / \mu_{c}$, which plays a role analogous to the magnetic field variable in a Ising system. $T_{c}$ and $\mu_{c}$ are defined in the thermodynamic limit, and the true second-order phase transition occurs for vanishing $t, r$ and $L^{-1}$ (and other couplings reaching their value at the fixed point, $\left\{g=g^{*}\right\}$ ).

For finite $L$, crossover effects become important. If the correlation length diverges as $\xi_{\infty} \sim t^{-\nu}$ at criticality, where $\nu$ is the corresponding critical exponent, in the case of $L^{-1} t^{-\nu} \gg 1$ the system is no longer governed by the critical fixed point and $L$ limits the growth of the correlation length, rounding all singularities [26]. If $L$ is finite, $\xi$ is analytic in the limit $t \rightarrow 0$, etc and one can draw scaling plots of $L / \xi$ vs. a given coupling $g$ for different values of $L$ to find that all curves cross at $g=g^{*}$ in this limit, which is a way to determine $g^{*}$. The critical temperature can also be determined in this fashion, since the curves will also cross at $t=t_{c}$. 
This scaling plot technique can be extended, taken to its full power for other quantities, such as correlation functions. An observable $X$ in a finite thermal system can be written, in the neighborhood of criticality, in the following form [16]:

$$
X(t,\{g\} ; \ell ; L)=L^{\gamma_{x} / \nu} f\left(t L^{1 / \nu}\right),
$$

where $\gamma_{x}$ is the bulk (dimension) exponent of $X$ and $\{g\}$ dimensionless coupling constants. The function $f(y)$ is universal up to scale fixing, and the critical exponents are sensitive essentially to dimensionality and internal symmetry, which will give rise to the different universality classes [19, 21]. To simplify the discussion, we ignore for the moment the chemical potential variable $r$. In principle, $f$ is a function of two scaling variables, though. Again, if one plots the scaled observable versus $t$, the curves should cross at $t=t_{c}$. Moreover, using the appropriate scaling variable, instead of $t$, all curves should collapse into one single curve if one is not far from the critical point. So, this technique can be applied to the analysis of observables that are directly related to the correlation function of the order parameter of the transition, such as fluctuations of the multiplicity of soft pions [9].

This is the idea of using full scaling plots, a method that has been proven to work even for tiny systems, to search for the critical endpoint of QCD in heavy ion data. Here, as in statistical mechanics searches for critical points, one can treat $T_{c}, \mu_{c}$ and the critical exponents as parameters in a scaling plot fit, the system size being provided by different centrality bins or by the number of participants in the collision. In fact, the scaling variable is defined up to $L$ - and $t$-independent multiplicative factors, so that the knowledge of the actual size of the system is not needed.

As mentioned previously, the correct scaling variable should measure the distance from the critical point, thereby involving both temperature and chemical potential, or their reduced versions $t$ and $r$. This produces a two-dimensional scaling function and makes the analysis of heavy ion data highly nontrivial. Phenomenologically, we adopt a simplification motivated by results from thermal models for the freeze-out region, connecting temperature and chemical potential. We can parametrize the freeze-out curve by $\sqrt{s_{N N}}$, and build our one-dimensional scaling variable from this quantity and the size of the system.

The range of sizes that can be accessed in heavy ion collisions over which FSS can be tested is limited. It is reasonable to assume that for a locally thermalized quark gluon plasma to form, the system needs to be several times the hadronic size (several $\mathrm{fm}$ ). The largest possible system will have a diameter of approximately $15 \mathrm{fm}$. Estimates from an analysis of HBT data from STAR indicate that from peripheral to central collisions, the system size changes by a factor of $3-4$ [22]. We plot the scaled observable vs $\left[\left(\mu-\mu_{c}\right) / \mu_{c}\right] L^{1 / \nu}$. The value of $L$ for the various centralities can be estimated from a Glauber Monte-Carlo model. For our study, the overlap area is calculated from $S_{\perp}=R_{A u}^{2}(\Theta-\sin \Theta)$ where $\Theta=2 \cos ^{-1}\left(b / 2 R_{A u}\right)$, where $R_{A u}$ is the nuclear radius for $A u$ and $b$ is the collision impact parameter. Then the length is taken to be $L=2 \sqrt{S_{\perp} / \pi}$.

A system of size $L_{1}$ can be compared to a system of size $L_{2}$ only when

$$
\mu_{1}-\mu_{c}=\left(\mu_{2}-\mu_{c}\right)\left(\frac{L_{2}}{L_{1}}\right)^{1 / \nu} .
$$

This constrains which $\mu$ values can be compared when testing for scaling by directly comparing data rather than by extrapolations. Since a central $A u+A u$ collision is about four times larger than a peripheral one $\left(L_{2}=4 L_{1}\right)$ and taking $\nu=2 / 3$, we find that a measurement in peripheral $\mathrm{Au}+\mathrm{Au}$ collisions at $\mu_{1}$ can be compared to a central collision at $\mu_{2}$ when $\mu_{1}-\mu_{c} \approx 8\left(\mu_{2}-\mu_{c}\right)$.

Data on various fluctuations have been measured at RHIC and the SPS over an energy range from $\sqrt{s_{N N}}=5$ $\mathrm{GeV}$ to $200 \mathrm{GeV}$ and the centrality dependence of $p_{T}$ fluctuations have been measured at RHIC for energies from 19.6 GeV to $200 \mathrm{GeV}$. This data provides an opportunity to look for evidence of finite-size scaling.

To search for scaling, we consider the correlation measure $\sigma_{p_{T}} /\left\langle p_{T}\right\rangle$ 23] scaled by $L^{-\gamma_{x} / \nu}$, according to Eq. 3. We consider the $p_{T}$ fluctuations $\sigma_{p_{T}}$ scaled by $\left\langle p_{T}\right\rangle$ to obtain a dimensionless variable ${ }^{1}$. We use the correlation data measured in bins corresponding to the $0-5 \%, 5-10 \%$, $10-20 \%, 20-30 \%, 30-40 \%, 40-50 \%, 50-60 \%$, and $60-70 \%$ most central collisions. We estimate the corresponding lengths $\mathrm{L}$ to be $12.4,11.1,9.6,8.0,6.8,5.6,4.5$, and 3.4 $\mathrm{fm}$. The exponent $\nu=2 / 3$ is determined by the Ising universality class of QCD and we consider values of $\gamma_{x}$ around 1 (ignoring small anomalous dimensions corrections). We also varied the value of $\gamma_{x}$ from 0.5 to 2.0 and found that changing $\gamma_{x}$ within this range does not improve the scaling behavior. Using a smaller value of $\gamma_{x}$ simply causes $\sigma_{p_{T}} /\left\langle p_{T}\right\rangle$ scaled by $L^{-\gamma_{x} / \nu}$ to drop more slowly with increasing centrality and increasing $\gamma_{x}$ causes this drop to happen more quickly.

In Figs. 1 1and 2 we plot $\sigma_{p_{T}} /\left\langle p_{T}\right\rangle$ scaled by $L^{-\gamma_{x} / \nu}$ vs $\mu$ for different system sizes, using data extracted from collisions at $\sqrt{s_{N N}}=19.6,62.4,130,200 \mathrm{GeV}$. We use the parameterization from Ref. [25] to convert from $\sqrt{s_{N N}}$ to $\mu$. If there is a critical point at $\mu=\mu_{\text {crit }}$, the curves for different sizes of the system should cross at this value of $\mu$. However, since the currently available data is restricted

\footnotetext{
1 The STAR collaboration has also published $p_{T}$ fluctuations using an alternative variable $\Delta \sigma_{p_{T}}[24]$. The results found using this variable are qualitatively equivalent to the ones for $\sigma_{p_{T}} /\left\langle p_{T}\right\rangle$.
} 


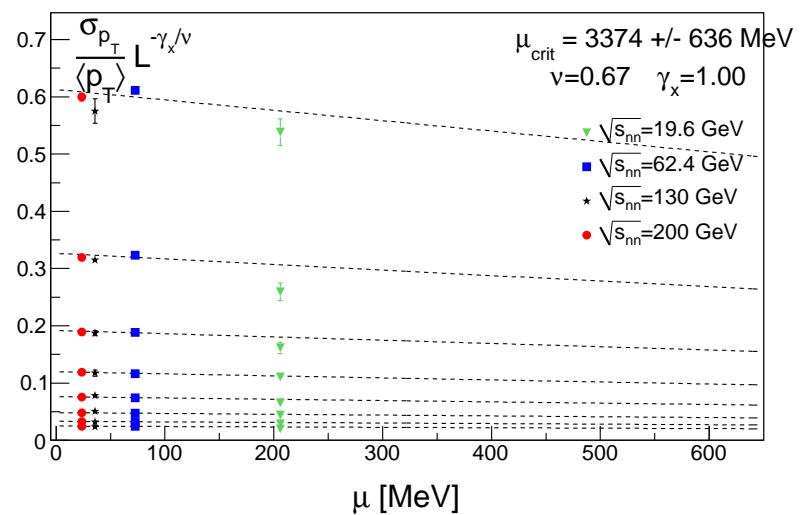

FIG. 1: Scaled $\sigma_{p_{T}} /\left\langle p_{T}\right\rangle$ vs $\mu$ for different system sizes, and with $\nu=2 / 3$ and $\gamma_{x}=1$. Data extracted from RHIC collisions at energies $\sqrt{s_{N N}}=19.6,62.4,130$, and $200 \mathrm{GeV}$ (linear fit, see text).

to not so large values of the chemical potential, one has to perform extrapolations using fits. The scaling function $f$ in Eq. 3 is expected to be smoothly varying around the critical point, so we fit the data corresponding to a given linear size $L$ to a polynomial, but constraining the polynomials to enforce the condition that all the curves cross at some $\mu=\mu_{\text {crit }}$, where $\mu_{\text {crit }}$ is an adjustable parameter in the fit. This clearly assumes the existence of a critical point. In Fig. 1 we use a linear fit. The approximate energy independence of $\sigma_{p_{T}} /\left\langle p_{T}\right\rangle$ along with the linear fit, leads to a very large $\mu$ value where the curves can cross $(\mu \sim 3 \mathrm{GeV})$. There's no reason however to assume a linear fit function, so in Fig. 2 we also try a second order polynomial. Using the a second order polynomial function for $f$ allows the curves from different system sizes to cross at a much smaller value of $\mu$. Based on this fit, we find that the data is consistent with a critical point at $\mu \sim 510 \mathrm{MeV}$ corresponding to a $\sqrt{s_{N N}}$ of $5.75 \mathrm{GeV}$.

The value estimated for $\mu_{\text {crit }}$ based on finite-size scaling of current data is highly dependent on the assumed functional form of $f$. The approximate energy independence of $\sigma_{p_{T}} /\left\langle p_{T}\right\rangle$ for a given $\mathrm{L}$, however, already indicates within the finite-size scaling assumption that the critical point should be at $\mu$ values well above those currently available. Based on finite-size scaling of $\sigma_{p_{T}} /\left\langle p_{T}\right\rangle$, one would not expect a critical point at $\mu<400 \mathrm{MeV}$. Having data for lower values of $\sqrt{s}$, i.e. higher values of chemical potential, as expected from the analysis of the Beam Energy Scan program at RHIC [27], one should be able to study full scaling plots of $\sigma_{p_{T}} /\left\langle p_{T}\right\rangle$ scaled by $L^{-\gamma_{x} / \nu}$ vs. $\frac{\mu-\mu_{\text {crit }}}{\mu_{\text {crit }}} L^{1 / \nu}$ without the need of long extrapolations. For the current set of data, these full scaling plots are still not very enlightening.

RHIC has also run at lower energies in order to search for a critical point in the Beam Energy Scan program. That data is currently being analyzed. Here we use the

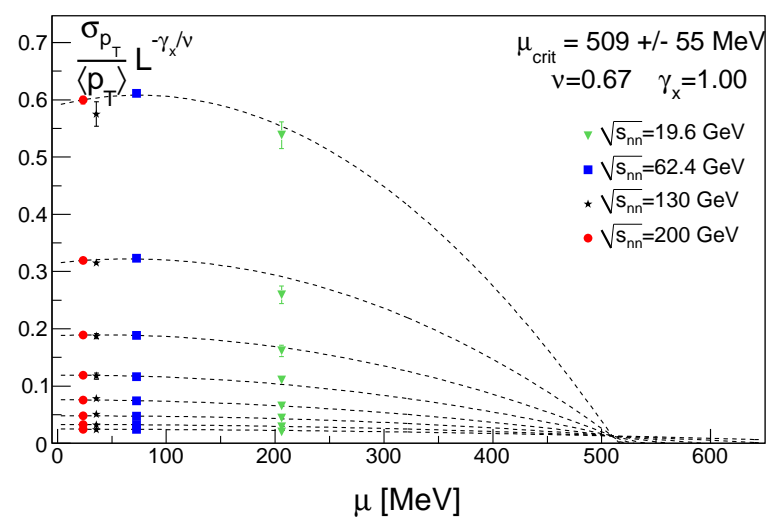

FIG. 2: Scaled $\sigma_{p_{T}} /\left\langle p_{T}\right\rangle$ vs $\mu$ for different system sizes, assuming $\mu_{\text {crit }}=509 \mathrm{MeV}$, which corresponds to a critical point at $\sqrt{s_{N N}}=5.75 \mathrm{GeV}$. Again, $\nu=2 / 3$ and $\gamma_{x}=1$. Data extracted from RHIC collisions at energies $\sqrt{s_{N N}}=19.6,62.4,130$, and $200 \mathrm{GeV}$ (second order polynomial fit, see text).

quadratic polynomial fit of STAR data (Fig. 2) and assume the critical point is at $509 \mathrm{MeV}$ to make predictions for $\sigma_{p_{T}} /\left\langle p_{T}\right\rangle$ at lower energies. The finite-size scaling scenario along with currently available data, allows us to predict the energy and system size dependence of fluctuations for any given values of $\mu_{\text {crit }}, \gamma_{x}$ and $\nu$. We show this expectation as a function of the number of participants, $N_{\text {part }}$, for three proposed beam energies: $11.5,7.7$ and $5 \mathrm{GeV}$ in Fig. 3 . Notice that the centrality dependence changes once one moves to the other side of the critical point. This is a condition enforced by finite-size scaling which provides a generic signal for having reached the first-order phase transition side of the critical point.

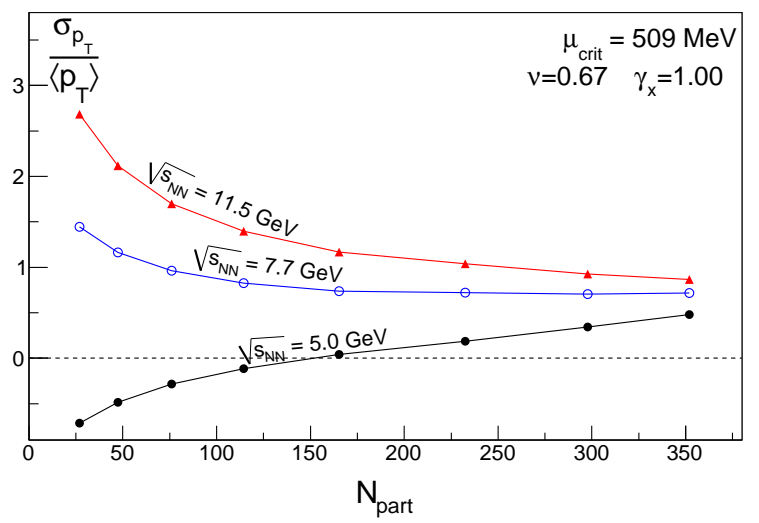

FIG. 3: The expected measurement of $\sigma_{p_{T}} /\left\langle p_{T}\right\rangle$ as a function of the number of participants at lower energies assuming the critical point is at $509 \mathrm{MeV}$ as extracted from the quadratic polynomial fit of STAR data.

Searching for the the critical endpoint in the phase di- 
agram for strong interactions is a remarkably challenging task. On one hand, lattice simulations can not provide the sort of guidance that is possible at zero density, where there is no sign problem, and effective models point to its existence, qualitatively, but yield very different quantitative predictions. On the other hand, heavy ion collision experiments probe limited regions of the phase diagram. Besides, they come with non-trivial background contributions that tend to blur the signatures provided by the non-monotonic behavior of observables built from correlation functions of the order parameter.

The fact that finite-size scaling prescinds from the knowledge of the details of the system under consideration, providing information about its criticality based solely on its most general features, makes it a very powerful and pragmatic tool for data analysis in the heavy ion collision search for the critical point. From a very limited data set in energy spam, we have used FSS to exclude the presence of a critical point at small values of the baryonic chemical potential, below $450 \mathrm{MeV}$. We have also used the scaling function to predict the behavior of data with system size at lower energies. We are looking forward to compare our predictions to the outcome of data analysis from the Beam Energy Scan program at RHIC.

We are grateful to M. Chernodub, T. Kodama, Á. Mócsy, K. Rajagopal, K. Redlich and M. Stephanov for fruitful discussions. This work was partially supported by CAPES-COFECUB (project 663/10), CNPq, FAPERJ and FUJB/UFRJ.

[1] A. Gopie and M. C. Ogilvie, Phys. Rev. D 59, 034009 (1999).

[2] A. Bazavov and B. A. Berg, Phys. Rev. D 76, 014502 (2007).

[3] O. Kiriyama and A. Hosaka, Phys. Rev. D 67, 085010 (2003); O. Kiriyama, T. Kodama and T. Koide, arXiv:hep-ph/0602086, L. M. Abreu, M. Gomes and A. J. da Silva, Phys. Lett. B 642, 551 (2006); L. M. Abreu, A. P. C. Malbouisson, J. M. C. Malbouisson, A. E. Santana, Nucl. Phys. B819, 127-138 (2009); L. M. Abreu, A. P. C. Malbouisson, J. M. C. Malbouisson, Phys. Rev. D83, 025001 (2011).

[4] J. Braun, B. Klein and H. J. Pirner, Phys. Rev. D 71, 014032 (2005); Phys. Rev. D 72, 034017 (2005); J. Braun, B. Klein, H. J. Pirner and A. H. Rezaeian, Phys. Rev. D
73, 074010 (2006).

[5] N. Yamamoto and T. Kanazawa, Phys. Rev. Lett. 103, 032001 (2009).

[6] L. F. Palhares, E. S. Fraga and T. Kodama, arXiv:0904.4830 [nucl-th]; PoS C POD2009, 011 (2009); J. Phys. G G37, 094031 (2010).

[7] C. Spieles, H. Stoecker and C. Greiner, Phys. Rev. C 57, 908 (1998).

[8] E. S. Fraga and R. Venugopalan, Physica A 345, 121 (2004).

[9] M. A. Stephanov, K. Rajagopal and E. V. Shuryak, Phys. Rev. Lett. 81, 4816 (1998); Phys. Rev. D 60, 114028 (1999).

[10] M. A. Stephanov, Phys. Rev. Lett. 102, 032301 (2009).

[11] M. Asakawa, S. Ejiri and M. Kitazawa, Phys. Rev. Lett. 103, 262301 (2009).

[12] B. Berdnikov and K. Rajagopal, Phys. Rev. D 61, 105017 (2000).

[13] M. A. Stephanov, Phys. Rev. D 81, 054012 (2010).

[14] M. E. Fisher, in Critical Phenomena, Proc. 51st Enrico Fermi Summer School, Varena, ed. M. S. Green (Academic Press, NY, 1972); M. E. Fisher and M. N. Barber, Phys. Rev. Lett. 28, 1516 (1972).

[15] E. Brezin, J. Physique 43, 15 (1982).

[16] E. Brezin and J. Zinn-Justin, Nucl. Phys. B 257, 867 (1985).

[17] P. de Forcrand, S. Kim, O. Philipsen, PoS LAT2007, 178 (2007); P. de Forcrand, O. Philipsen, JHEP 0811, $012(2008)$.

[18] J. Cleymans, H. Oeschler, K. Redlich and S. Wheaton, Phys. Rev. C 73, 034905 (2006).

[19] C. Domb and J. L. Lebowitz (Eds.), Phase Transitions and Critical Phenomena (Academic, London, 1987), vols. 8 and 11.

[20] J. L. Cardy, Scaling and Renormalization in Statistical Physics (Cambridge, 1996).

[21] D. Amit, Field Theory; The Renormalization Group and Critical Phenomena (World Scientific, 2005).

[22] J. Adams et al. [STAR Collaboration], Phys. Rev. C 71, 044906 (2005).

[23] J. Adams et al. [STAR Collaboration], Phys. Rev. C 72, 044902 (2005).

[24] J. Adams et al. [STAR Collaboration], J. Phys. G 34, 451 (2007).

[25] J. Cleymans, H. Oeschler, K. Redlich and S. Wheaton, Phys. Rev. C 73, 034905 (2006).

[26] N. Goldenfeld, Lectures on Phase Transitions and the Renormalization Group (Addison-Wesley, New York, 1992).

[27] M. M. Aggarwal et al. [STAR Collaboration], arXiv:1007.2613 [nucl-ex]. 